\title{
Migrations- und kontaktinduzierte Mischvariante Jugendlicher im Spannungsfeld zwischen medialer Stilisierung und alltäglichem Gebrauch
}

\author{
Migration- and contact-derived youth language variant \\ in tension between media and primary style use
}

\author{
Anna DASZKIEWICZ ${ }^{1}$ \\ Ateneum-Szkoła Wyższa w Gdańsku
}

\begin{abstract}
Zusammenfassung
Der vorliegende Beitrag wendet sich der migrations- und kontaktinduzierten Mischvariante Jugendlicher und junger Erwachsener zu. Hierbei liegt der Fokus sowohl auf die ursprüngliche, primäre, ethnolektal gefärbte Sprachvariante, als auch deren medial-stilisierte Form. Der Beitrag veranschaulicht die Tendenzen im gesprochenen Gegenwartsdeutsch, sensibilisiert für dessen Transgression sowie Übertragung auf das mediale Terrain und lässt das Spannungsfeld zwischen medialer Stilisierung und alltäglichem Gebrauch erfahren.

Schlüsselwörter: der primäre, sekundäre, tertiäre Ethnolekt, das medial verbreitete Gegenwartsdeutsch, das Standarddeutsch, die Abweichung vom Standarddeutschen, deutschund andersstämmige Jugendliche und/oder junge Erwachsene, gesprochenes Deutsch in stark besiedelten, urbanen Gebieten
\end{abstract}

\footnotetext{
Abstract

1 https://orcid.org/0000-0003-3745-8528

Ateneum-Szkoła Wyższa w Gdańsku, Wydział Neofilologiczny

a.daszkiewicz@ateneum.edu.pl
}

This paper addresses the language youth variant triggered by migration and contacts. The focus is put on both the primary form of the so-called ethnolect as well as the media representation 
and is transgression to everyday register. The paper shows tendencies in contemporary spoken German and allows recognition of media-presented ethnolect and its primary form, and shows similarities and differences between the two of them.

Keywords: primary, secondary, tertiary ethnolect, media-spread contemporary German, standard German language, deviations from the language norm, youth and young adults of German and other roots, spoken German in strongly urbanised city centres

\section{Einleitung}

Es unterliegt keinem Zweifel, dass die wachsende Mobilität, sowie Lernbereitschaft bei jüngeren Generationen den Rückgang des Dialektgebrauchs herbeiführt. Der besagte Trend lässt sich in den letzten Jahrzehnten insbesondere in Ballungszentren der deutschen Großstädte beobachten, wo regionale Dialekte an ihrer Aussagekraft deutlich verloren haben. Nichtsdestotrotz scheint ein anderes Sprachregister, gemeint ist das ethnolektal gefärbte Gegenwartsdeutsch oder aber auch der Ethnolekt des Deutschen, diese Lücke erfolgreich geschlossen zu haben. Damit wird eine migrations- und kontaktinduzierte Sprechvariante bezeichnet, die sich stark vom Standarddeutsch unterscheidet und inzwischen auch von den Sprechern der deutschen Standardsprache gebraucht wird.

Als wichtigster Vertreter des Ethnolekts ist der Türkenslang (Androutsopoulos 2000, 2001, 2003, 2007, Auer 2003) oder die Kanaksprak (Zaimoğlu 1995, 1997) in das Bewusstsein sowie die Sprechgewohnheiten der jungen Erwachsenen getreten. Hierbei beschränkt sich die die Ethnolektforschung in bundesdeutschem Kontext auf wenige Autoren. Dies ist darauf zurückzuführen, dass Ethnolektsprecher den Ethnolekt nicht konsequent verwenden und beim Sprechen unterschiedliche Sprachregister zur Anwendung bringen. So ist der Ethnolekt nicht nur schwer ab- oder aber auch eingrenzbar, sondern auch stets veränderbar. Während im Fall von Dialekten des Deutschen eine Einteilung nach geographischen Gesichtspunkten möglich ist, wird mit dem Ethnolektgebrauch die Zugehörigkeit zu einer bestimmten Gruppe/Szene oder gesellschaftlichen Schicht zum Ausdruck gebracht. Kurzum identifizieren sich die Ethnolektsprecher mit ihrer gesellschaftlichen Gruppe oder Clique durch ihren Ethnolekt und neigen auf diese Weise zur Abgrenzung von den deutschen Standardsprechern. Hierbei werden Ethnolektsprechern stereotype Eigenschaften zugeschrieben. Demnach sind ihnen ein provozierendes Auftreten sowie die Gewaltbereitschaft unterstellt. Solche Unterstellungen sind allerdings auch bei den Dialekten des Deutschen zu 
finden. Während Schwaben beispielsweise als fleißig gelten, stuft man Sachsen als langsam ein. Diese stereotypen Merkmale von Dialekten werden in vielen Kunstformen- ob Kabarett, Theater oder Fernsehen - der Parodie einzelner Personen oder ganzer Gruppen genutzt. Einer besonderen Beliebtheit erfreut sich nach wie vor die mediale Verbreitung des Ethnolekts. „Comedystars, Schauspieler und Kabarettisten machen sich den deutsch-türkischen Ethnolekt zu Nutze: Pro 7 sendet Erkan und Stefan, der SWR etabliert Taxi Sharia; Bülent Cylan, Django Asyl oder Mundstuhl bringen die Kanaksprak auf Kabarettund Comedybühnen“ (Androutsopoulos 2000: 1).

\section{Türkenslang als Ethnolekt des Deutschen}

Auer und Androutsopoulos drücken mithilfe des Begriffs Ethnolekt eine Abweichung des Deutschen aus, „die von einer oder mehreren ethnischen Gruppen verwendet und mit diesen Gruppen assoziiert wird“ (Auer 2003: 2)². Es handelt sich dabei um ein Ausländerdeutsch, das zwar in mehreren ethnischen Gruppen vorhanden ist, dennoch besonders von der deutschtürkischen Szene dominiert wird. Es fällt auf „[...], dass in multiethnischen Stadtteilen von Hamburg der Gebrauch türkischer Routinen Bestandteil des üblichen jugendlichen Sprachgebrauchs ist" (Androutsopoulos 2000: 4.). Wie bereits zuvor erwähnt, lässt sich der ethnolektal gefärbte Sprechstil nur schwer in seiner Gesamtheit erfassen und weist starke Veränderungen auf. Nichtsdestotrotz gibt es einen prototypischen „Nährboden“ (Androutsopoulos 2001: 4.), der die Identifikation des betreffenden Ethnolekts gerade für Außenstehende leicht überschaubar macht. Den Ethnolekt kennzeichnen und so verraten nämlich lautliche, lexikalische bzw. grammatikalische Merkmale, sowie Eigenschaften des soziokulturellen Kontextes, in denen er jeweils zum Vorschein kommt. Der typische Ethnolektsprecher ist demnach ein männlicher Jugendlicher türkischer Abstammung, der in Deutschland aufgewachsen, der Ausbildung kaum Interesse schenkt, lokal eingeschränkte Bindungen pflegt und sich in einem subkulturellen Gesellschaftsmilieu bewegt (Ebd.).

\footnotetext{
2 Vgl. auch Androutsopoulos, Jannis: Ethnolektale Entwicklungen im Sprachgebrauch Jugendlicher. Beitrag in der Sektion 3 „Gesprochene Sprache in regionaler und sozialer Differenzierung“ auf dem 10. internationalen Germanistenkongress, Universität Wien, 10.-15. September 2000, S. 1.
} 


\subsection{Die Stufen des Ethnolekts}

Was die sprachliche Manifestation des Ethnolekts angeht, werden von Auer (2003: 2 ff.) drei Stufen unterschieden. Der primäre Ethnolekt gilt als die Standardvarietät, die sich in Alltagsgesprächen zwischen zwei oder mehreren deutsch-türkischen Sprechern vollzieht. Der primäre Ethnolekt wird von mehrsprachigen Jugendlichen erworben, in Alltagssituationen vollzogen und nicht - wie im Falle des sekundären Ethnolekts - dank der medialen Aufbereitung bekannt und folglich auch nur zitiert. Der sekundäre Ethnolekt ist eher ein stark verzerrtes, je nach der Mediengattung stilisiertes und grundsätzlich ironisch eingesetztes Konstrukt. Es finden sich hier zwar Merkmale des primären Ethnolekts wieder, doch durch die stilistische Verstärkung sowie lexikalische Neugestaltungen entsteht eine durchaus neue Varietät. Als Beispiel gelten die Medienmacher wie z.B. Mundstuhl, die als Deutschstämmige keinen echten Kontakt zu den Ethnolektsprechern haben und dessen primäre Variante als ihnen nicht zu eigene Sprechweise zitieren, was Auer (2003: 3) als sprachliche „Transgression“ definiert. Übernehmen die deutschen Standardsprecher die medial stilisierte Sprechvariante und gebrauchen sie situationsspezifisch, handelt es sich um einen tertiären Ethnolekt. Dieser dient allerdings im Generellen nicht der Kommunikation, sondern offenbart einen eher provokativen Charakter, indem damit Zwecke wie eine scherzhafte Anmache, gezielte Angriffe oder Darstellung der eigenen Medienkenntnis realisiert werden. Nun aber können sich auch Personen mit einem ausschließlich deutschen Hintergrund den primären Ethnolekt aneignen, falls sie sich an direkten Gesprächen mit bilingualen Verwendern des Deutsch-Türkischen beteiligen. Dies findet bei "gemischtethnischen Jugendlichennetzwerken in den deutschen Großstädten“" (Ebd.) statt.

\subsection{Spezifik des medial-sekundären Ethnolekts}

Es sei an dieser Stelle betont, dass zur Zeit neben der primären Ethnolektvariante auch seine medial stilisierte und aufgrund ihrer häufigen Präsenz auf Kabarett- und Comedybühnen dem nicht-einheimischen Publikum überhaupt erst bekannte Variante in den vermehrten Fokus der soziolinguistischen Forschung gerät. Gemeint ist der sekundäre bzw. medial-sekundäre Ethnolekt (nach Auer 2003), der in der vorliegenden Arbeit in Bezug auf seine Gemeinsamkeiten und Differenzen zu dem primären Ethnolekt veranschaulicht und so noch ausführlicher charakterisiert wird. 


\subsubsection{Spezifik des medial-sekundären Ethnolekts im Hinblick} auf Gemeinsamkeiten zum primären Ethnolekt

\begin{tabular}{|c|c|}
\hline & $\begin{array}{l}\text { Spezifik des medial-sekundären Ethnolekts } \\
\text { (im Hinblick auf Gemeinsamkeiten zum primären Ethnolekt) }\end{array}$ \\
\hline 1. & $\begin{array}{l}\text { Die beiden Ausprägungen des Ethnolekts beziehen sich auf Strategien verbaler } \\
\text { und paraverbaler Kommunikation Jugendlicher anderer Muttersprachen in } \\
\text { großstädtischen Vierteln mit hohem Migrantenanteil. }\end{array}$ \\
\hline 2. & $\begin{array}{l}\text { Die beiden Ethnolektformen fördern/arbeiten für ein Verständnis von Sprache } \\
\text { als nicht lediglich ein Herkunftsmarker, sondern eher eine wichtige Ressource } \\
\text { zur Herausbildung der »Peergruppen-Identität« (im Sinne von Androutsopoulos/ } \\
\text { Georgakopoulou 2003: 9). }\end{array}$ \\
\hline 3. & $\begin{array}{l}\text { Konstituierung der sozialen Identität: In den beiden Ethnolektrepräsentationen } \\
\text { dient Sprache also als unabdingbares Mittel der intentionalen Selbstkreation als } \\
\text { Individuum und als Teil einer Gruppe zum einen, der Positionierung/ } \\
\text { Stellungnahme gegenüber der somit ausgedrückten Selbstdarstellung zum } \\
\text { anderen. Kurzum: Mit und mittels des primären und medial-sekundären } \\
\text { Ethnolekts wird die soziale Identität/die soziale Positionierung der Sprechenden/ } \\
\text { die Eigen-/und Fremdstilisierung konstituiert und thematisiert. So betrachtet, } \\
\text { kommt den beiden Ethnolektformen die Funktion der kommunikativen } \\
\text { Verbindung und Abgrenzung zu. }\end{array}$ \\
\hline 4. & $\begin{array}{l}\text { Kontextualisierung der sozialen Identität: Die beiden Ausprägungen des } \\
\text { Ethnolekts erfordern einen klar umrissenen situativen Kontext, in dem sie ihre } \\
\text { vollumfängliche Bedeutung entfalten und im Nachhinein einer sprachbezogenen } \\
\text { Untersuchung sowie Auswertung (im Hinblick auf deren Verwendung und } \\
\text { Funktion) unterzogen werden können. }\end{array}$ \\
\hline 5. & $\begin{array}{l}\text { Sowohl der primäre als auch der medial-sekundäre Ethnolekt setzt die Kenntnis } \\
\text { der Vielfalt an Sprachvarianten sowie deren Zielsetzung voraus (die beiden } \\
\text { Ethnolekt-Ausprägungen sind auf ein deutschlandkompetentes Publikum } \\
\text { zugeschnitten). }\end{array}$ \\
\hline 6. & $\begin{array}{l}\text { Die beiden Ethnolekt-Manifestationen verweisen andererseits aber auch auf } \\
\text { einen kompetenten Umgang mit verschiedenen Ausdrucksweisen (eine kreative } \\
\text { Stilbastelei), die durch Prozesse der Enkodierung, Übernahme, Transformation, } \\
\text { Adaptation sowie (prosodischen) Imitation von verschiedenen kulturellen und } \\
\text { sprachlichen Ressourcen gekennzeichnet ist. }\end{array}$ \\
\hline 7. & $\begin{array}{l}\text { Wiederkehrende Stil-Montagen (Bricolagen und Bricolage-Muster) in einer } \\
\text { realen oder fiktiven Peergruppe initiieren und stimulieren Wortneubildungen } \\
\text { und Phraseologismen etc. }\end{array}$ \\
\hline 8. & $\begin{array}{l}\text { Kontextualisierung von Spaß: Die beiden Ethnolektformen treten in einer } \\
\text { vertrauten Sprechsituation in Erscheinung und sind auf den mehr oder weniger } \\
\text { individuellen Spaß ausgerichtet (sie werden auf jeden Fall in stark emotional } \\
\text { geladenen, meist spaßigen, scherzhaften Momenten in Gang gesetzt). }\end{array}$ \\
\hline
\end{tabular}




\begin{tabular}{|l|l|}
\hline 9. & $\begin{array}{l}\text { Hohe Relevanz und Brisanz: Sie sind mittlerweile ein unabdingbares } \\
\text { Ausdrucksmittel der gegenwärtigen Popkultur und somit Gegenstand } \\
\text { offentlichen und wissenschaftlichen Interesses. }\end{array}$ \\
\hline 10. & $\begin{array}{l}\text { Sie stehen dem schulisch (institutionell) geforderten Prinzip der sprachlichen } \\
\text { Reinheit oder dem Einsprachenmodell entgegen. }\end{array}$ \\
\hline 11. & $\begin{array}{l}\text { Für deren Gesamtbild arbeiten, wie bereits oben angedeutet, verbale und } \\
\text { paraverbale Ausdrucksmittel. Nicht nur sprachlich markierte Standardferne, } \\
\text { sondern auch Proxemik, Gestik, Bewegungsformen sowie Präferenzen bezüglich } \\
\text { der Kleidung (Bomberjacken, Springerstiefel, Goldketten) machen den } \\
\text { ethnolektalen Sprechstil leicht identifizierbar. }\end{array}$ \\
\hline 12. & $\begin{array}{l}\text { Die beiden Ethnolektformen stellen hoch ausdifferenzierte (nicht klar festgelegte } \\
\text { und abgeschlossene) Subsysteme dar, die in Bezug auf Verwendung und Funktion } \\
\text { von Sprachmerkmalen sogar bei ein und demselben Sprecher (der primäre } \\
\text { Ethnolekt) oder je nach der Mediengattung, dem gesellschaftlichen und } \\
\text { politischen Kontext sowie individuellen Verhältnis der Medienmacher zu } \\
\text { dem/der reproduzierten Code/Stimme (der medial-sekundäre Ethnolekt) stark } \\
\text { variieren können. }\end{array}$ \\
\hline 13. & $\begin{array}{l}\text { Kurzum fungieren sie als eine aber nicht einzige und wie bereits oben } \\
\text { hervorgehoben, situationsangemessen verwendbare Sprachvariante/sprachliche } \\
\text { Ressource, die dem Diktat der sozio-politischen Lage und gesellschaftlichen } \\
\text { Erwartung unterworfen ist. }\end{array}$ \\
\hline 14. & $\begin{array}{l}\text { Mittels der beiden Ethnolekt-Ausprägungen werden Alltagsthemen eines } \\
\text { bestimmten sozialen Milieus sowie dessen Sichtweisen auf die } \\
\text { Mehrheitsgesellschaft (vermeintliche Sichtweisen der Mehrheitsgesellschaft auf } \\
\text { die Minderheitengruppen mit einbezogen) aufgegriffen und im vertrauten Kreis } \\
\text { oder im komischen Rahmen verarbeitet. }\end{array}$ \\
\hline
\end{tabular}

\subsubsection{Spezifik des medial-sekundären Ethnolekts im Hinblick auf Unterschiede} zum primären Ethnolekt

\begin{tabular}{|l|l|}
\hline & $\begin{array}{l}\text { Spezifik des medial-sekundären Ethnolekts } \\
\text { (im Hinblick auf Unterschiede zum primären Ethnolekt) }\end{array}$ \\
\hline 1. & $\begin{array}{l}\text { Mediale Stilisierungen des Straßenethnolekts haben reale sprachliche } \\
\text { Gegebenheiten in stark besiedelten urbanen Wohngebieten abzubilden und zu } \\
\text { interpretieren. Zentral ist hier die Haltung des Sprechenden zu der zitierten } \\
\text { Stimme. }\end{array}$ \\
\hline 2. & $\begin{array}{l}\text { Da sich an der medialen Umsetzung gewöhnlich keine (bzw. nur ausnahmsweise) } \\
\text { Träger des primären Ethnolekts beteiligen, kommen ethnolektale } \\
\text { Sprachmerkmale nie (oder nur selten) in ihrer authentischen Form auf die } \\
\text { Bühne/den Bildschirm. Dadurch kann die Verwendung des medial-sekundären } \\
\text { Ethnolekts mitunter zur peinlichen Inszenierung und Anbiederung werden. } \\
\text { Alles in allem handelt es sich bei einer derartigen (medial fundierten) }\end{array}$ \\
\hline
\end{tabular}




\begin{tabular}{|c|c|}
\hline & $\begin{array}{l}\text { hahmung (»Usurpierung« einer Stimme, die einem aus ethnischen oder } \\
\text { ialen Gründen nicht eigen ist [»Transgression« im Sinne von Dirim/Auer 2004: } \\
\text { um eine Kunst/Pseudo- oder massenmedial vermittelte Scherz/Spaßvarietät; } \\
\text { ich: eine Kommunikationsform der neuen Medien. }\end{array}$ \\
\hline 3. & $\begin{array}{l}\text { Aufgrund hyperbolischer, übertriebener Ausdrucksweisen ist der medial- } \\
\text { sekundäre Ethnolekt also als Scherzvarietät, und zwar mit einem nicht } \\
\text { interaktiven (rhetorischen) Charakter wahrzunehmen. }\end{array}$ \\
\hline 4. & $\begin{array}{l}\text { Wie oben teilweise nahegelegt, wecken mediales und öffentliches Interesse } \\
\text { vorwiegend „dialogisch-performative Formen der Mündlichkeit“ (Reershemius/ } \\
\text { Ziegler 2015: 241), die zudem vor Obszönität und Vulgarität nicht } \\
\text { zurückschrecken, medial übersteigert und dicht angewandt sind (vgl. Bell/Gibson } \\
\text { 2011: 557). }\end{array}$ \\
\hline 5. & $\begin{array}{l}\text { Selektionsverfahren ethnolektal geprägter semantischer und semiotischer } \\
\text { Eigenschaften (die einen ethnolektalen Merkmale werden „getilgt“/,gelöscht“ } \\
\text { (vgl. Androutsopoulos 2007), die anderen hingegen rekurrent verwendet und so } \\
\text { „ikonisiert“ [ebd.]) steht einer kompletten/komplexen Beschreibung der } \\
\text { Kommunikations- und Handlungspraktiken in ethnisch gemischten } \\
\text { Jugendgruppen entgegen, sprich: lässt die Heterogenität des ursprünglichen } \\
\text { ethnolektalen Sprechstils ausblenden. }\end{array}$ \\
\hline 6. & $\begin{array}{l}\text { Daher stellt der medial-sekundäre Ethnolekt ein strukturell deutlich } \\
\text { eingeschränktes, aber dennoch durchsichtiges, leicht abrufbares und } \\
\text { konnotierbares sprachliches Gerüst dar. }\end{array}$ \\
\hline 7. & $\begin{array}{l}\text { Der medial-sekundäre Ethnolekt stellt demnach eine soziolinguistische } \\
\text { Indexikalisierung dar, die die Zuordnung der Individuen zu sozialen Gruppen } \\
\text { oder Schichten ermöglicht und begünstigt. Im Gegensatz zum ursprünglichen } \\
\text { Ethnolekt wird mittels des medial reproduzierten Etnolekts nicht nur die } \\
\text { Selbstverortung im Migrantenumfeld, sondern auch die Kontextualisierung und - } \\
\text { profilierung des Fremden vollzogen. }\end{array}$ \\
\hline 8. & $\begin{array}{l}\text { Der medial-sekundäre Ethnolekt sorgt für die Integration standardferner } \\
\text { Sprechweisen Jugendlicher deutscher und nicht-deutscher Abstammung } \\
\text { (mittelbar aber auch für die Integration der Träger des primären Ethnolekts), die } \\
\text { gesamtgesellschaftlich eher negativ assoziiert und bewertet werden: Da } \\
\text { medieninduzierte Sprachkonstrukte meistens im komischen Rahmen in den } \\
\text { Fokus geraten, sind sie in der Regel nicht in derselben Form provozierend, } \\
\text { drastisch und vulgär. }\end{array}$ \\
\hline 9. & $\begin{array}{l}\text { Andererseits kann somit effizient zur Herstellung von weiteren Stereotypen } \\
\text { beigetragen werden: Karikieren, Persiflieren und Ironisieren von ethnisch } \\
\text { gemischten Gruppen und deren sprachlichen Präferenzen, die mittels medialer } \\
\text { Sprachkonstrukte bewirkt werden, dienen aufgrund der nachgewiesenen } \\
\text { positiven Wirkung des Lachens zwar Entlastungszwecken, können jedoch nicht } \\
\text { ausschließlich als ein „harmloses Spiel“ mit als exotisch empfundenen } \\
\text { Sprachelementen ausgelegt und somit verharmlost werden (vgl. Dirim/Auer }\end{array}$ \\
\hline
\end{tabular}




\begin{tabular}{|c|c|}
\hline & $\begin{array}{l}\text { 2004: 222). Die hohe Verdichtung und Verfremdung von medial fundierten } \\
\text { Sprechweisen gepaart mit einer zielbewussten Distanzierung zu Sprechen des } \\
\text { realen Straßenethnolekts deutet (im Falle der Fremdstilisierung im Besonderen) } \\
\text { ziemlich genau auf fremdenfeindliche Stimmungen in der Mehrheitsgesellschaft } \\
\text { hin. }\end{array}$ \\
\hline 10 . & $\begin{array}{l}\text { Mittels medialer Stilisierungen erfolgt nicht nur die Integration, sondern auch } \\
\text { Interaktion verschiedener Ausdrucksformen. Parallelisieren und Kontrastieren } \\
\text { von sprachlichen Varianten (stilistisches Schillern) ist pädagogisch und } \\
\text { methodisch-didaktisch von kaum zu überschätzender Bedeutung. }\end{array}$ \\
\hline 11. & $\begin{array}{l}\text { Die Besonderheit des medial-sekundären Ethnolekts ergibt sich im Übrigen aus } \\
\text { seinen Charakter und Funktion: Massenhafte Verbreitung und kontinuierliches } \\
\text { Aufrollen von standardfernen Sprachpraktiken schließen es vehement aus, den } \\
\text { ethnolektalen Sprechstil lediglich ,unter sich“ (bei engen Freundschaften) und in } \\
\text { Abgrenzung zu der Mehrheitsgesellschaft zu verwenden. Mediale Umsetzung des } \\
\text { Ethnolekts hat einen gruppenexternen und nicht -internen Charakter, wirkt } \\
\text { zwar jugendspezifisch aber nicht unbedingt jugendtypisch (eher } \\
\text { generationsübergreifend). Doch der medial-sekundäre Ethnolekt hat ein } \\
\text { möglichst großes Publikum anzusprechen und für wirtschaftliche Ziele zu } \\
\text { gewinnen. }\end{array}$ \\
\hline 12. & $\begin{array}{l}\text { Der medial-sekundäre Ethnolekt verfügt also über eine hohe soziale Reichweite: } \\
\text { Er wird ungeachtet von Alter, Bildungsgang und gesellschaftlicher Position der } \\
\text { ZuschauerInnen in alle Haushalte transportiert. Somit hat er einen ungleich } \\
\text { höheren Einfluss auf die Umgangssprache als weite Teile der Literatur. }\end{array}$ \\
\hline 13. & $\begin{array}{l}\text { „Mediale Stilisierungen haben das Potential, soziale und semiotische Moden } \\
\text { spezifischer sozialer Gruppen zu popularisieren und damit } \\
\text { Sprachveränderungsprozesse zu verstärken“ (Reershemius/Ziegler 2015: 241). }\end{array}$ \\
\hline 14. & $\begin{array}{l}\text { Wie bereits vorstehend angesprochen, werden mittels des medial-sekundären } \\
\text { Ethnolekts sprachbezogene Kreuzungs- und Mischungsprozesse sowie } \\
\text { 'Normverstöße' provoziert. Der medial-sekundäre Ethnlekt initiiert, verstärkt, } \\
\text { popularisiert und beschleunigt den phonetisch-prosodisch, morphologisch- } \\
\text { syntaktisch und lexikalisch-semantisch ausgerichteten Wandel. }\end{array}$ \\
\hline 15. & über Sprachgebrauch und Sprachwandel an. \\
\hline
\end{tabular}

Nun aber soll die oben präsentierte, von mir engmaschig gesammelte und dargelegte Auflistung der Spezifika des medial-stilisierten Ethnolekts keinerlei den Eindruck verschaffen und hinterlassen, als würde sich die besagte Form in höherem Maße als der primäre Ethnolekt der Beliebtheit der Sprachforschung erfreuen. Denn seit gut ein paar Jahrzehnten wird den Gesprächen zwischen türkischstämmigen Zuwanderern und ihren in Deutschland geborenen und großgezogenen Nachkommen sowie den zwischen deutsch- und andersstämmigen Jugendlichen und jungen 
Erwachsenen in stark besiedelten, urbanen Gebieten zugehört. Stellvertretende Ausdrücke werden dann aufgenommen und der sprachwissenschaftlichen Analyse übergeben. Hierbei versucht man stets die in großen Ballungszentren entstandene Sprachvarietät mit dem Standarddeutschen $\mathrm{zu}$ vergleichen, d.h. auf deren Abweichungen und Gemeinsamkeiten zum Standarddeutschen hinzuweisen.

\section{3. Überblick über den Forschungsstand}

Die Forschung in Bezug auf den primären Ethnolekt, der in dieser Arbeit näher gebracht wird, ist zurzeit noch gut überschaubar. Aus anderen Sprachräumen gäbe es vergleichbare Entwicklungen, diese sollen hier jedoch unberücksichtigt bleiben. Stattdessen wird hier auf die wichtigsten Forschungsergebnisse im deutschen Sprachraum verwiesen. Hierbei werden der besagten migrationsund kontaktinduzierten Mischvariante Jugendlicher nicht nur unterschiedliche Bezeichnungen/Namen gegeben, sondern auch Eigenschaften zugeschrieben. Dies schlägt sich in der unten präsentierten Charakteristik nieder. Die hier gesammelten und veranschaulichten Ergebnisse offenbaren die Tendenzen im gesprochenen Gegenwartsdeutsch und wurden dem Werk der meist stellvertretenden Forschern auf diesem Gebiet entnommen: dementsprechend dem Werk von Heike Wiese (2009, 2012), Diana Marossek (2016) und Uwe Hinrichs (2013). 


\begin{tabular}{|c|c|c|}
\hline $\begin{array}{l}\text { - Veränderungen im } \\
\text { - „Kiezdeutsch» (W } \\
\text { »Kurzdeutsch» (M }\end{array}$ & $\begin{array}{l}\text { PARALLELEN } \\
\text { chenen Deutsch } \\
\text { Multi Kulti Deutsch« (Hinrichs) oder } \\
\text { ) sind mit der Jugendsprache schlecht } \\
\end{array}$ & \\
\hline & N & \\
\hline & $\downarrow$ & $\Delta$ \\
\hline $\begin{array}{l}\text { „Kiezdeutsch«/»Kiezsprache« } \\
\text { (Heike Wiese) } \\
\text { - ein multiethnischer Dialekt } \\
\text { (»Turbodialekt») } \\
\text { - ein kreatives und produktives } \\
\text { Sprachsystem (weiblicher und } \\
\text { männlicher) Jugendlicher anderer } \\
\text { Muttersprache in dicht besiedelten } \\
\text { urbanen Räumen } \\
\text { - ein Konglomerat von Kontaktsprache } \\
\text { und Jugendsprache (sowie lokalen } \\
\text { und dialektalen Einflüssen) } \\
\text { - ein landübergreifendes, mehr } \\
\text { generationen- als altersspezifisches } \\
\text { Sprachphänomen } \\
\text { - beschränkt auf bestimmte soziale } \\
\text { Gruppen sowie informelle Kontexte } \\
\text { - seine Abweichungen dem } \\
\text { Standarddeutsch gegenüber } \\
\text { resultieren nicht aus willkürlich } \\
\text { getroffenen, fehlerhaften } \\
\text { Entscheidungen, sondern verweisen } \\
\text { auf in sich stimmige und bereits im } \\
\text { System des Deutschen angelegte } \\
\text { Entwicklungen/ } \\
\text { Entwicklungsmöglichkeiten } \\
\text { Interferenzen aus den Muttersprachen } \\
\text { Andersstämmiger beziehen sich } \\
\text { hauptsächlich auf die Lexik und } \\
\text { teilweise das Lautsystem; sie werden } \\
\text { allesamt an die Regeln der deutschen } \\
\text { Grammatik und Aussprache } \\
\text { angepasst } \\
\text { bildet keine Grundlage für } \\
\text { Sprachverarmung oder Sprachverfall, } \\
\text { kein »defizitäres«, ॥falsches« Deutsch } \\
\text { - Ergebnis kreativer Produktiviät, } \\
\text { Sprachspielerei (Montage) } \\
\text { - diese kontaktinduzierten } \\
\text { Sprachveränderungsprozesse sind } \\
\text { spannend und zeugen von einer } \\
\text { besonderen sprachlichen Integration } \\
\text { multi- und monoethnisch geprägter } \\
\text { junger Menschen } \\
\text { die vorstehend veranschaulichten } \\
\text { „Kiezdeutsch«-Charakteristika werden } \\
\text { anhand empirischer Daten aus den } \\
\text { Regionen Berlin-Kreuzberg und Berlin- } \\
\text { Hellersdorf aufgestellt }\end{array}$ & $\begin{array}{l}\text { "MKurzdeutsch» } \\
\text { (Diana Marossek) } \\
\text { - ein kreatives und produktives } \\
\text { Sprachsystem weiblicher und } \\
\text { männlicher, multi- und } \\
\text { monoethnischer Jugendlicher und } \\
\text { Erwachsener in ganz Berlin } \\
\text { - wird ebenso von deutschen } \\
\text { Muttersprachler/inne/n gesprochen, } \\
\text { die in keinem Kontakt zu ethnisch } \\
\text { geprägten Gruppen stehen (nicht nur } \\
\text { in dicht besiedelten urbanen } \\
\text { Räumen) } \\
\text { - ein Konglomerat von Stadtsprache } \\
\text { und Migrantensprache (insbesondere } \\
\text { "Türkendeutsch«) } \\
\text { - ein generationenübergreifendes } \\
\text { Sprachphänomen } \\
\text { - beschränkt weder auf ethnische bzw. } \\
\text { soziale Gruppen noch informelle } \\
\text { Kontexte } \\
\text { - seine Abweichungen dem } \\
\text { Standarddeutsch gegenüber } \\
\text { resultieren nicht aus willkürlich } \\
\text { getroffenen, fehlerhaften } \\
\text { Entscheidungen, sondern verweisen } \\
\text { auf in sich stimmige und bereits im } \\
\text { System des Deutschen und des } \\
\text { Türkischen angelegte Entwicklungen/ } \\
\text { Entwicklungsmöglichkeiten } \\
\text { - Interferenzen aus den } \\
\text { Muttersprachen Andersstämmiger } \\
\text { beziehen sich auf die Lexik und } \\
\text { Morphosyntax, teilweise auch das } \\
\text { Lautsystem; dabei wird auch das } \\
\text { Lokalkolorit (das Berlinerische) } \\
\text { manifest } \\
\text { - zu seiner Verbreitung tragen } \\
\text { traditionelle und neue Medien } \\
\text { erheblich bei } \\
\text { - diese kontaktinduzierten } \\
\text { Sprachveränderungsprozesse sind } \\
\text { spannend und dauerhaft } \\
\text { - die vorstehend veranschaulichten } \\
\text { "Kurzdeutsch«-Charakteristika } \\
\text { werden anhand empirischer Daten } \\
\text { aus sämtlichen Stadtvierteln in Berlin } \\
\text { aufgestellt }\end{array}$ & $\begin{array}{l}\text { „Multi Kulti Deutsch«/ } \\
\text { »Neudeutsch» } \\
\text { (Uwe Hinrichs) } \\
\text { - sein Motor ist die } \\
\text { Mehrsprachigkeit, aber auch die } \\
\text { Globalisierung und Anglisierung } \\
\text { (Modernisierung) der Welt } \\
\text { - besteht aus Simplifizierungen und } \\
\text { Reduktionen, die auf } \\
\text { Interferenzfehler aus den } \\
\text { Herkunftssprachen } \\
\text { Hinzugezogener aber auch eine } \\
\text { mangelnde Bereitschaft zur } \\
\text { Korrektur bei den Nicht- } \\
\text { Hinzugezogenen verweisen und } \\
\text { eine Art kommunikativen (die } \\
\text { gemeinsame Verständigung } \\
\text { sichernden) Kompromisses sind } \\
\text { - signalisiert das Verschwimmen } \\
\text { von Grenzen zwischen »falschem« } \\
\text { und »richtigem« Deutsch } \\
\text { (»Hopping«) sowie einen } \\
\text { kollektiven und nicht bewussten } \\
\text { Prozess (»Trampelpfad«) } \\
\text { - Interferenzen aus den } \\
\text { Muttersprachen Andersstämmiger } \\
\text { beziehen sich auf alle } \\
\text { Sprachbereiche } \\
\text { - beschränkt zwar auf bestimmte } \\
\text { soziale Gruppen aber nicht nur } \\
\text { informelle Kontexte } \\
\text { - bildet die Grundlage für } \\
\text { Sprachverarmung und } \\
\text { Sprachverfall } \\
\text { - die vorstehend veranschaulichten } \\
\text { „Multi Kulti Deutsch«- } \\
\text { Charakteristika werden durch } \\
\text { keine Feldforschung oder } \\
\text { empirischen Daten bestätigt }\end{array}$ \\
\hline
\end{tabular}

Abbildung 1: Drei Modelle, die die Tendenzen im gesprochenen Gegenwartsdeutsch charakterisieren 
Wie vorstehend präsentiert, unterscheiden sich die Sichtweisen der Sprachforscher auf die Tendenzen im gesprochenen, migrationsund kontaktinduzierten Gegenwartsdeutsch enorm. Im Zusammenhang damit wird der primäre Ethnolekt als ein kreatives und produktives Sprachsystem zum einen, eine Sprachverarmung oder sogar ein Sprachverfall zum anderen wahrgenommen. Zudem wird mal auf seinen generationenspezifischen sowie informellen Charakter, mal seinen generationenübergreifenden Umfang und Gebrauch hingedeutet. All dies mag nur jegliche Kontroversen um das besagte sprachliche Phänomen betonen.

\section{Schlussbemerkungen}

Im vorliegenden Beitrag wird auf das stets wachsende Interesse an den Tendenzen im gesprochenen Gegenwartsdeutsch hingewiesen. Es wird hier nicht nur der Überblick über den Forschungsstand verschafft, sondern auch für die mitunter gegensätzliche Bewertung der sprachlichen Änderungen (selbst unter Sprachwissenschaftlern) sensibilisiert. Darüber hinaus wird dem Nährboden für die erwähnten Änderungen, sprich Abweichungen vom Standarddeutsch genauer hingesehen und bemerkt, dass die migrationsund kontaktinduzierte Sprechweise nicht lediglich ,auf das eigene Stadtviertel“ einzuschränken ist. Das abweichend klingende Sprachregister wird nämlich gerne aufgegriffen, medial verbreitet und in den eigenen Ausdruck mit einbezogen (der primäre, sekundäre und tertiäre Ethnolekt nach Auer 2003). Selbst wenn sich der medial-sekundäre Ethnolekt von dem primären unterscheidet und zunächst überzogen wirkt, spielen zahlreiche Gemeinsamkeiten auf einen künftigen Trend im gesprochenen Deutsch ab, was letztendlich der Aufmerksamkeit der Linguisten kaum entgehen kann.

\section{BIBLIOGRAFIE}

Androutsopoulos, J. (2000). Ethnolektale Entwicklungen im Sprachgebrauch Jugendlicher. Beitrag in der Sektion 3 „Gesprochene Sprache in regionaler und sozialer Differenzierung" auf dem 10. internationalen Germanistenkongress, Universität Wien, 10.-15. September. 2000.

Androutsopoulos, J. (2001). From the streets to the screens and back again: On the mediated diffusion of variation patterns in contemporary German. In: LAUD Linguistic Agency, Series A: No. 522. Universität Essen.

Androutsopoulos, J. (2003). jetzt speak something about italiano. Sprachliche Kreuzungen im Alltagsleben. In: Erfurt, J. (Hrsg.), „Multisprech“: Hybridität, Variation, Identität. (S. 79-109). Oldenburg. 
Androutsopoulos, J. \& Georgakopoulou, A. (2003). Discourse constructions of youth identities. Amsterdam: John Benjamins.

Androutsopoulos, J. (2007). Ethnolekte in der Mediengesellschaft. Stilisierung und Sprachideologie in Performance, Fiktion und Metasprachdiskurs. In: Fandrych, Ch. \& Reiner, S. (Hrsg.), Standard, Variation und Sprachwandel in germanischen Sprachen. (S. 113-155). Tübingen: Narr.

Androutsopoulos, J. (2007). Neue Medien - neue Schriftlichkeit?, Mitteilungen des Deutschen Germanistenverbandes, 1 (07), 72-97.

Auer, P. (2003) „Türkenslang“ - ein jugendsprachlicher Ethnolekt des Deutschen und seine Transformationen. In: Häcki-Buhofer, A. (Hrsg.), Spracherwerb und Lebensalter. (S. 255-264). Tübingen/Basel.

Bell, A. \& Gibson, A. (2011). Staging language: An introduction tot he sociolinguistics of performance. Journal of Sociolinguistics 15. 55-72.

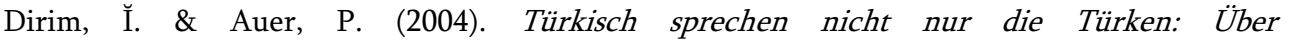
die Unschärfebeziehung zwischen Sprache und Ethnie in Deutschland. Berlin.

Hinrichs, U. (2013). Multi Kulti Deutsch. Wie Migration die deutsche Sprache verändert. München: C.H. Beck Verlag.

Marossek, D. (2016). Kommst du Bahnhof oder hast du Auto? Warum wir reden, wie wir neuerdings reden. Berlin: Hanser Verlag.

Reershemius, G. \& Ziegler, E. (2015). Sprachkontaktinduzierte jugendkulturelle Stile im DaFUnterricht: Beispiele aus dem Film Fack ju Göhte. In: Imo, W. \& Moraldo, S. (Hrsg.): Interaktionale Sprache im DaF-Unterricht. (S. 241-274). Tübingen: Stauffenburg Verlag.

Wiese, H. (2009). Grammatical innovation in multiethnic urban Europe: New linguistic practises among adolescents, Lingua 119, 782-806.

Wiese, H. (2012). Kiezdeutsch. Ein neuer Dialekt entsteht. München: CH Beck.

Zaimoğlu, F. (1995). Kanak Sprak. 24 Mißtöne vom Rande der Gesellschaft. Hamburg: Rotbuch.

Zaimoğlu, F. (1997) Abschaum. Die wahre Geschichte von Ertan Ongun. Hamburg: Rotbuch. 